\title{
Zur Erinnerung
}

\section{Zum 500.Todestag Nikolaus' von Kues (1401-1464)}

Vor 500 Jahren, am 11. August 1464, starb in Todi Nikolaus von Kues. Im Rahmen der Feiern, die in seinem Geburtsort Kues, zum Teil im heute noch bestehenden, von ihm für alte Leute gestifteten St.-Nikolaus-Hospital stattfanden, widmet Dr. Paul LüTh, Arzt und Schriftsteller in Rengshausen (Kassel), dieser berühmten Figur des deutschen Humanismus eine interessante Arbeit ${ }^{1}$. Die erstaunlich vielseitige, stark ausgeprägte, menschlich warme Persönlichkeit des Cusanus - Jurist, Philosoph und Mann der Praxis, Forscher zugleich und Entdecker von Plautus- und TAcitus-Handschriften sowie ehrlich nach Reformen strebender Theologe, Bischof, später Kardinal, päpstlicher Legat, Freund und Berater des Pius II. Piccolomini - wird in dieser Arbeit fest umrissen. Neben der weniger bekannten politischen und diplomatischen Tätigkeit, neben den damals revolutionär anmutenden Gedankengängen (so lehnte er z. B. vor GaliLeI die geozentrische Lehre des Planetensystems ab), sei hier besonders auf den Dialog «Der Laie» hingewiesen. Wie Kollege Lüth treffend dazu bemerkt, besteht kein Zweifel, «daß Nikolaus Naturwissenschaft in unserem Sinne der quantitativen Methodik meinte. Er demonstriert das ausführlich an der Medizin. Von den damit beginnenden Atmungs-, Puls- und Gewichtsmessungen und ihren Vergleichen würde man nicht nur zu bisher unbekannten Graden der Krankheit, sondern auch zu Graden der Gesundheit kommen; ebenso würde sich die Verschiedenheit der Altersstufen dadurch neu einsehen lassen.»

Anhand einer kritischen Analyse von Leben und Werken des Cusanus weiß Dr. Lüth eine Reihe negativer Urteile von Professor JAspers über diesen Humanisten überzeugend - so will mir scheinen - und nicht ohne Humor zu entkräften.

Dr.med.René Kaech, Basel

Die feierliche Cusanus -Tagung vom 9. bis 12. August 1964 in Kues an der Mosel bot ein imponierendes Bild von der menschlichen, wissenschaftlichen und religiöskirchlichen Bedeutung des großen Denkers, die einen großen Teil der CusanusForscher vereinte. Auch Raymund Klibansky, Montreal, mit Ernst HoffMANN $\dagger$, Heidelberg, zusammen der erste Herausgeber der großen (noch nicht vollendeten) kritischen Ausgabe der Schriften des Cusaners, war zugegen und sprach über «Nachwirken und Wiederaufleben des Nikolaus von Kues». Für den Naturwissenschaftshistoriker waren von besonderer Bedeutung die Vorträge: W.Dupré, Wien: Die Idee einer neuen Logik bei Nikolaus von Kues; J. Meurers,

${ }^{1}$ Deutsches Ärzteblatt, Ärztliche Mitteilungen 61 (1964) 31-32, 1733-1740. 
Wien: Nikolaus von Kues und die Entwicklung des astronomischen Weltbildes; N.Stuloff, Mainz: Nikolaus von Kues in der Mathematikgeschichte.

Aus der neuen Cusanus-Literatur sind von Interesse: A.KrchnaK, Die Herkunft der astronomischen Handschriften und Instrumente des Nikolaus von Kues, Mitt. Forsch.beitr. Cusanus-Ges. 3 (1963) 109-180; E. Meuthen, Nikolaus von Kues, Skizze einer Biographie (Aschendorffsche Verlagsbuchhandlung, Münster (Westfalen) 1964; R.Haubst, Nikolaus von Kues und die moderne Wissenschaft, Kleine Schriften der Cusanus-Gesellschaft, Trier $1963 . \quad H . F$.

«Wenn die denkende Seele mit höchster Sehnsucht getrieben wird, zu unterscheiden und zu wissen - wenn sie dahin gelangt, daß sie die Ursache ihrer so großen Sehnsucht in sich selbst, nämlich in ihrer Unterscheidungskraft, sieht, dann hat sie jedenfalls in sich das Wissen von dem Geber dieser Sehnsucht. Und sie kann nichts erstreben, was sie nicht in sich selbst sieht. Denn was könnte der, der sich nach Wissen sehnt, noch weiteres verlangen, wenn das Verursachte das Wissen um seine Ursache in sich erschaut. Denn dann hat er den Wesensgrund und die Kunst seiner Erschaffung in sich; und das ist die Erfüllung und Vollendung aller Wissenschaft.» Nikolaus von Kues (Vom Globusspiel, herausgegeben von Gerda von Brednow, F. Meiner, 1952). 